\title{
Effects of Lipid Overload on Heart in Metabolic Diseases
}

\section{(ㄷ) (i) (우) $\Theta$}

\author{
Authors \\ An $\operatorname{Yan}^{1}{ }^{*}$, Guinan $X_{i e}{ }^{*}$, Xinya Ding ${ }^{1}$, Yi Wang ${ }^{1}$, Liping Guo ${ }^{2}$
}

Affiliations

1 Tianjin University of Traditional Chinese Medicine, Tianjin, China

2 Tianjin Academy of Traditional Chinese Medicine, Tianjin, China

Key words

lipid metabolism, cardiac energy metabolism, metabolic diseases, metabolic syndrome, type 2 diabetes, diabetic cardiomyopathy

received 16.08 .2021

accepted after revision $\quad 29.10 .2021$

\section{Bibliography}

Horm Metab Res 2021; 53: 771-778

DOI $10.1055 / a-1693-8356$

ISSN 0018-5043

(c) 2021. The Author(s).

This is an open access article published by Thieme under the terms of the Creative Commons Attribution-NonDerivative-NonCommercial-License, permitting copying and reproduction so long as the original work is given appropriate credit. Contents may not be used for commecial purposes, or adapted, remixed, transformed or built upon. (https://creativecommons. org/licenses/by-nc-nd/4.0/)

Georg Thieme Verlag KG, Rüdigerstraße 14,

70469 Stuttgart, Germany

\author{
Correspondence \\ Yi Wang \\ Institute of Traditional Chinese Medicine \\ Tianjin University of Traditional Chinese Medicine \\ 300193 Tianjin \\ China \\ Tel: +86-22-59596555 \\ wangyi@tjutcm.edu.cn \\ Liping Guo \\ Tianjin Academy of Traditional Chinese Medicine \\ 300120 Tianjin \\ China \\ Ipgtjn@163.com
}

\section{ABSTRACT}

Metabolic diseases are often associated with lipid and glucose metabolism abnormalities, which increase the risk of cardiovascular disease. Diabetic cardiomyopathy (DCM) is an important development of metabolic diseases and a major cause of death. Lipids are the main fuel for energy metabolism in the heart. The increase of circulating lipids affects the uptake and utilization of fatty acids and glucose in the heart, and also affects mitochondrial function. In this paper, the mechanism of lipid overload in metabolic diseases leading to cardiac energy metabolism disorder is discussed.

\section{Introduction}

Metabolic diseases (MetDs), which include metabolic syndrome, pre-diabetes, and type 1 and 2 diabetes, are a series of pathological conditions characterized by abnormal glucose use in the body. Multiple clinical studies have shown that metabolic syndrome and prediabetes both increase the risk of cardiovascular disease and allcause mortality [1-5], and that cardiac function and structure have changed in prediabetes patients [6-9]. In fact, obesity, insulin resistance, and hyperglycemia are independent risk factors for the development of diabetic cardiomyopathy (DCM), which is the leading cause of death in MetDs [10,11]. DCM, mainly manifested as cardiac systolic dysfunction, is an important factor in the development of heart failure [12].
Cardiac energy metabolism disorder is considered to have a very important effect on the function and structure of the heart. Under normal physiological conditions, the use of fatty acids and glucose by myocardium is strictly regulated. However, under pathological conditions, the metabolism of fatty acids and glucose in cardiomyocytes changes significantly. The increase of fatty acid metabolism caused mitochondrial damage, leading to serious heart damage [13]. Both clinical and animal studies have reported increased uptake and utilization of lipids and accumulation of fat in heart in metabolic syndrome/diabetes [14-16]. The purpose of this review is to explain the role of lipids in cardiac energy metabolism and to elucidate a series of pathologic changes that occur in the heart when lipid overload occurs.

An Yan and Guinan Xie contributed equally to this work. 


\section{Cardiac Energy Metabolism in the Healthy}

\section{Heart}

The heart converts chemical energy from fatty acids and glucose into mechanical energy for actin-myosin interactions. Due to the higher productivity fatty acids provide $70 \%$ of the ATP needed by the heart. Fatty acids are the main energy fuel for the heart, followed by glucose [17]. Interestingly, in the immature stage of cardiomyocyte the main energy comes from glycolysis $[18,19]$. As cardiomyocytes continue to differentiate and mature, they gradually begin to rely on oxidative phosphorylation for energy. When the heart is saturated with energy requirements, excess glucose is stored as glycogen. When the heart is low on energy, stored glycogen can be disintegrated as fuel to produce ATP [20]. As the result of highly dependence on fatty acid explains why chronic changes in fatty acid metabolism can have profound effects on cardiac function. In conclusion, the selection, utilization, and storage of myocardial fuel is a complex physiological process.

Cardiac energy metabolism mainly includes three parts: substrate utilization, oxidative phosphorylation, and high-energy phosphate metabolism [21]. If the energy produced does not meet the heart's needs, it can gradually lead to a decline in heart function and even heart failure [21]. Mitochondrial ATP supply to myocardium requires the support of two important processes: metabolic fuel uptake and mitochondrial structural and functional integrity. A defect in either link can quickly lead to a decline in cardiac function. Under the stimulation of insulin and myocardial mechanical contraction, fatty acid translocase FAT/CD36 and GLUT4 are transported from endoplasmic reticulum to the cell membrane to uptake most of the fatty acids and glucose into the cardiomyocytes [22]. Fatty acid acyl-coenzyme A (Fa-CoA) is synthesized by fatty acids under the action of Fa-CoA synthetase but cannot enter the mitochondria directly. Fa-CoA is first transferred to carnitine by carnitine palmitoyltransferase-1 (CPT-1), and then acylcarnitine enter the mitochondria directly, where it is converted back to Fa-CoA by carnitine palmitoyltransferase-2 (CPT-2) in the inner membrane of mitochondria. After entering the mitochondria, Fa-CoA is oxidized to acetyl-CoA through $\beta$-oxidation [23]. Glucose produces pyruvate, which then enters the mitochondria and enters the tricarboxylic acid (TCA) cycle. Acetyl CoA is the confluence of fatty acid and glucose metabolism and can enter the TCA cycle freely to produce reducing agents $\mathrm{NADH}$ and $\mathrm{FADH}_{2}$, and transfer electrons to the mitochondrial respiratory chain for oxidative phosphorylation [24]. Because enzymes involved in fatty acids and glucose metabolism inhibit the oxidation of the other fuel, neither fuel disintegrates at the same time [25].

On the other hand, mitochondria consume oxygen and ADP to synthesize ATP through oxidative phosphorylation of complexes I, II, III, and IV located in the mitochondrial intima, and at the same time use energy released by electron transport process to establish proton gradient across the intima [26, 27]. NADH and succinic acid formed in the TCA cycle are oxidized by complexes I and II, transferring electrons to ubiquinone. Under the action of complex III, ubiquinone transfers electrons to cytochrome $C$, and finally electrons transfer to complex IV to reduce oxygen to water [27, 28]. During electron transport, protons are pumped out of the mitochondrial matrix and create an electrochemical gradient on the mitochondrial lining. $\mathrm{F}_{0} \mathrm{~F}_{1}$-ATP synthase uses the proton gradient to generate free energy to power the synthesis of ATP from ADP [28] (॰ Fig. 1).

\section{Lipid Overload Resulted in Increased FAT/ CD36 Translocation to the Cell Membrane and Decreased GLUT4}

Although the mechanism is unclear, it is widely believed that lipid overload causes insulin increase. Since CD36 is more sensitive to insulin than GLUT4, it induces CD36 transfer from endoplasmic reticulum to sarcomembrane when insulin is increased in circulation. In addition, a new study showed that excess lipids also induced CD36 translocation through upregulation of PKC $\zeta$ activity and TBC1D1 phosphorylation [29]. This change results in the persistence of CD36 in the sarcomembrane, which in turn promoted the myocardial fatty acid uptake rate [30]. Although there was an increase in CD36 expression in the sarcomere, there was no change in total expression. This was due to the transfer of CD36 from the endoplasmic reticulum to the sarcomere rather than increased protein expression [31, 32]. An increase in fatty acid transport was observed under a high-fat diet induced model, apparently prior to insulin resistance. Before insulin resistance, CD36 translocation increased under the action of lipids and insulin, while GLUT4 translocation is not affected [33]. Increased fatty acid oxidation and rapid intracellular lipid accumulation lead to increased protein acetylation levels and lipid intermediates, which inhibit insulin signaling at multiple levels [34-36]. When insulin resistance occurs, Akt2-mediated GLUT4 translocation is inhibited, leading to a decrease in glucose transport rate and a further increase in fatty acid uptake [33].

However, inhibition of fatty acid transport does not improve heart function. A study has shown that the specific ablation of CD36 on cardiomyocytes severely obstructs the utilization of fatty acids, leading to the over-dependence of the heart on glucose for energy and further accelerating the development of heart failure [37]. Therefore, the heart can only function properly when the use of fatty acids and glucose is in balance. Otherwise, too much fatty acids or glucose can cause heart damage. Re-balancing substrate uptake is an effective therapy to correct cardiometabolic disorder [38]. It has also been shown that supplementing or promoting non-lipid myocardial metabolic substrates can improve the state of high-fat induced cardiac energy metabolism imbalance. In vitro and in vivo studies have demonstrated that specific amino acid supplementation (lysine, leucine, arginine) reinternalized CD36 to the endosomes by regulating the mTORC1-v-ATPase axis, reduces myocardial lipid uptake and reverses/prevents lipid accumulation [39].

\section{Excessive Fatty Acid Utilization Affects Myocardial Energy Efficiency}

Clinical data show that a significant decrease in myocardial glucose uptake in T2DM patients is associated with predominant lipid utilization [40]. In db/db mouse heart, oxidation of fatty acids relative to glucose was reported to have increased by $64 \%$ [41], providing more than $90 \%$ of the heart's ATP [42]. The flow of glycolysis and glycogen decomposition decreased by a factor of two and three [41]. 


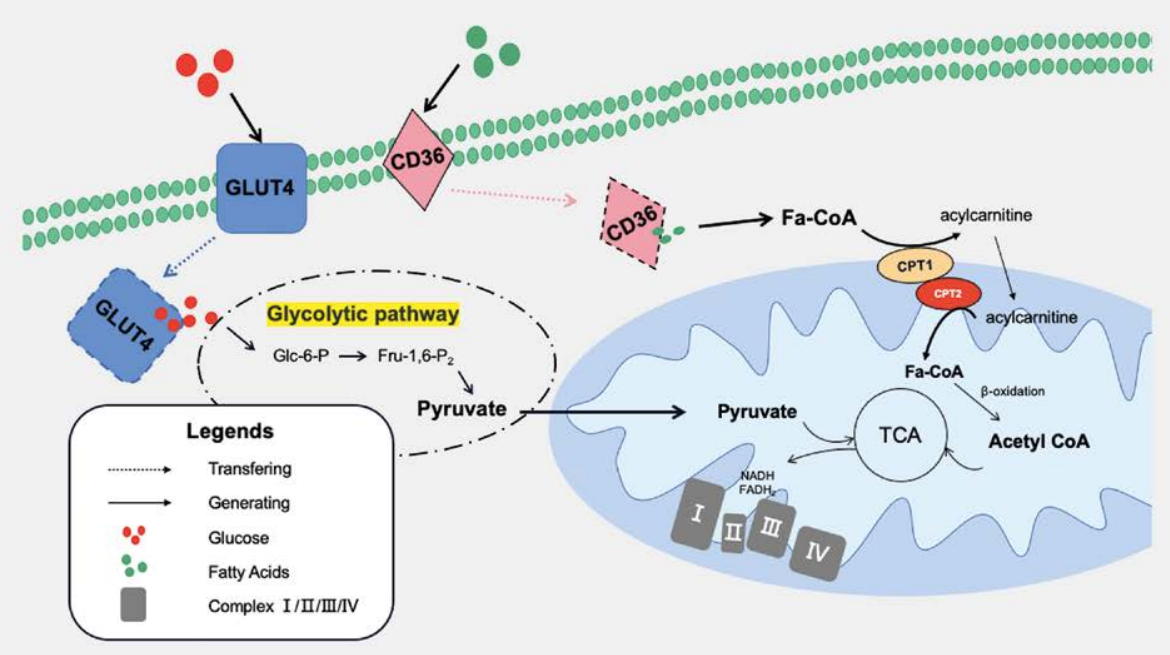

- Fig. 1 Uptake and utilization of fatty acids and glucose in the healthy heart.

The effects of fatty acid oxidation on the heart have been elucidated in several reviews $[24,43,44]$, which will not be repeated in this review. Circulating lipid overload promotes CD36-mediated myocardial fatty acid uptake and reduces GLUT4 translocation. This leads to increased uptake of fatty acids and reduced glucose in the myocardium. On the other hand, the expression of PPAR $\alpha$ and enzymes involved in $\beta$-oxidation were upregulated by fatty acid stimulation, which further promoted the increase of myocardial fatty acid oxidation flux [45-47]. Due to the increased dependence of cardiomyocytes on fatty acids, the ratio of cardiac fatty acid oxidation to glucose oxidation increases, which foiled the development of DCM. Glutaredoxin 3 (Grx3) is generally thought to protect cardiomyocytes from oxidative stress-induced damage by regulating REDOX states in mammals $[48,49]$. But a new study found that Grx3 is also an important part of regulating cardiac metabolism. Grx3 regulates the balance of cardiac energy metabolism by up-regulating the expression of proteins related to fatty acid uptake, transport and oxidation and down-regulating the expression of proteins related to glucose uptake and utilization [50].

Fatty acids are more productive than glucose, producing more ATP per carbon molecule, but they also require more oxygen [23]. In diabetes, increased oxidation of fatty acids causes the heart muscle to consume scarce oxygen more quickly. When the situation develops further - hypoxia becomes more serious - fatty acid oxidation gradually decreases, and excessive dependence on fatty acids for energy leads to myocardial systolic dysfunction and myocardial hypertrophy, which is the energy metabolism process in the development of DCM. Prostaglandin E receptor (EP4) is one of the receptors of prostaglandin E2, which is widely expressed in cardiomyocytes. Recent studies have shown that EP4 changes CD36 expression in HFD-induced DCM by regulating FOXO1/CD36 signaling axis and improves cardiac fatty acid metabolism and ATP production [51].

\section{Effect of Lipid Overload on Mitochondrial Function}

Mitochondria, as biological energy metabolic centers in eukaryotic organisms, provide the site for a variety of biochemical processes, including oxidative phosphorylation (OXPHOS), the tricarboxylic acid cycle, fatty acid $\beta$-oxidation, calcium treatment, and heme biosynthesis [52]. Mitochondrial structural integrity is critical to its function and vitality, but mitochondrial damage has been observed in a variety of metabolic diseases. Mild diastolic/systolic dysfunction occurs in prediabetic heart, accompanied by impaired mitochondrial function, including impaired mitochondrial respiration and ATP production, and decreased mitochondria, etc. [53, 54]. When myocardial insulin resistance or diabetes occurs, by increasing mitochondrial division and reducing mitochondrial fusion, reducing mitochondrial oxidative capacity, increasing ROS production and mitochondrial decoupling, mitochondrial function is impaired, leading to mitochondrial damage, myocardial cell death, and cardiac dysfunction [55-58].

Chronic elevated blood glucose provides an environment for myocardial ischemia and hypoxia, resulting in impaired mitochondrial function of cardiomyocytes [59]. As myocardial contraction and membrane potential ion pump require energy from mitochondria, cardiomyocytes are particularly sensitive to hypoxic injury $[60,61]$. Therefore, previous studies have suggested that the main cause of mitochondrial function impairment in metabolic diseases is oxidative stress injury caused by ischemia and hypoxia, but the damage of lipid overload to mitochondria cannot be ignored. Under normal conditions, mitochondrial structural integrity maintains a steady state of synthesis and degradation under the control of a variety of mitochondrial fusion and fission proteins. Mitofusin 1 (MFN1), Mitofusin 2 (MFN2), and protein Optic Atrophy 1(OPA1) are the key GTPases of mitochondrial fusion, among which the first two are responsible for mitochondrial outer membrane fusion $[62,63]$, and OPA1 regulates mitochondrial inner membrane fusion 
[64]. Excessive fatty acid uptake in cardiomyocytes can reduce the expression of mitochondrial fusion genes MFN1, MFN2, and OPA1, resulting in mitochondrial structure remodeling, and inhibit the activity of respiratory chain complex and oxidative phosphorylation [65]. Dynamin-related protein 1 (Drp1) is a large GTP enzyme that mediates mitochondrial division. Drp1 acts on the outer membrane of mitochondria through receptor proteins and activates downstream signaling pathways through multiple phosphorylation sites to promote mitochondrial fission [52]. Recent studies have found that excess lipids activate Drp1 through acetylation and increase mitochondrial translocation, leading to myocardial cell dysfunction and death [66]. In vitro experiments also confirmed that the increase of FA in myocardium significantly reshaped the mitochondrial network, resulting in the accumulation of slender mitochondria with reduced diameter in myocardium cells, and induced mitochondrial dynamic changes mediated by the post-translational modifications of mitochondrial protein, DRP1 and OPA1, leading to mitochondrial dysfunction [67].

\section{Cardiolipotoxicity}

Excessive lipid accumulation in the non-adipose cells of the cardiovascular system leads to cell dysfunction and cell death, a process known as lipotoxicity. When excess lipids are deposited in the heart, they can lead to myocardial cell apoptosis and cardiac systolic dysfunction [68]. Excessive fatty acids can selectively absorb and store fatty acids by upregulating GSK-3 $\alpha$ and phosphorylating PPAR $\alpha$ at Ser280 of its ligand binding domain, promoting fat accumulation, which is the basis for the development of the metabolic disease lipotoxic cardiomyopathy [69].

As a storage form of fat, triacylglycerol (TAG) itself has no direct lipid toxicity to myocardium. However, the lipid intermediates produced in the process of TAG synthesis and decomposition are believed to be responsible for the lipid toxicity, as well as post-translational modification of proteins.

\section{Diacylglycerol (DAG)}

Lipid intermediates are a class of signaling molecules produced during the intracellular accumulation of fatty acid, which regulate the energy supply and survival of cardiomyocytes. DAG is an important lipid intermediate in TAG synthesis and decomposition. It is widely believed that the activation of DAG- protein kinase $C$ (PKC) induces insulin resistance (details will be described in the next section). Inhibiting the amount of DAG in myocardium may be an effective treatment. DAG acyltransferase (DGAT) is a key enzyme in the synthesis of DAG from 3-phosphoglyceride and acyl-CoA. A study has shown that partial inhibition of DGAT activity increases cardiac fatty acid oxidation but does not affect PPAR $\alpha$ signal transduction or cardiac systolic function. Complete inhibition of DGAT activity can eliminate cardiac lipid accumulation induced by high fat diet without adverse effects on basic cardiac function [70].

\section{Ceramide}

Ceramides are the most widely studied sphingolipids involved in cardiac lipotoxicity. Studies have shown that ceramide levels in circulation and myocardium are positively associated with the risk of cardiovascular events and mortality [71, 72]. When triglyceride stores are also saturated, acyl-CoA enters the ceramide biosynthesis pathway. Excessive ceramide can induce insulin resistance $[35,73]$, regulate the translocation of CD36 to the muscle membrane, inhibit the uptake of glucose and amino acids, reduce mitochondrial efficiency and slow lipolysis by blocking activation of hormonesensitive lipase (HSL) in cardiomyocytes [74].

\section{Long-chain acyl-CoA}

Upon entry into cardiomyocytes, long-chain fatty acids bind with CoA molecules to form long-chain acyl-CoA (LCACoA). The direct lipid toxicity of LCACoA to cardiomyocytes has yet to be determined, and current reports indicate that LCACoA has a dual role in myocardial mitochondria. Palmitoyl CoA (PCoA) can induce the loss of $\Delta \Psi$ m outside the mitochondria and thus affect mitochondrial function [75]. PCoA can also limit the shuttle of ADP and ATP on the mitochondrial membrane by inhibiting the activity of ADP/ATP carriers on the mitochondrial membrane [76, 77]. However, during ischemia, ATP can be prevented from entering mitochondria for hydrolysis, which is a protective mechanism during ischemia [78]. However, in diabetes, the protective membrane potential of PCOA is inhibited, leading to increased ATP hydrolysis rate in diabetic heart during ischemia [78].

\section{Post-translational modifications(PTMs) of proteins}

PTMs of proteins refers to a covalent process that a protein undergoes during or after translation, which can be regarded as a switch on which proteins function. Palmitoylation of proteins is the reversible linking of palmitate molecules to cysteine residues under the action of enzymes. Depending on the hydrophobicity of palmitate, the modified protein can be localized to specific submembrane. However, lipid overload can lead to aberrant/excessive palmitoylation of proteins, which negatively affects insulin signaling [79]. For example, hyperpalmitylation of PKC $\varepsilon$ leads to downregulation of insulin receptor expression and decreased insulin sensitivity [80]. CD36 hyperpalmitylation promotes fatty acid uptake by increasing the number of CD36 on the cell membrane [81].

Acetylation of proteins is also a reversible modification. Since lysine acetyltransferase that mediates protein acetylation is produced by acetyl CoA, increased fatty acid metabolism leads to an increase in protein acetylation levels [34]. In the insulin signaling pathway, acetylation of Akt and its upstream regulator, phosphoinositol-dependent kinase 1 (PDK1), blocks insulin signaling and inhibits translocation of GLUT4 [82, 83].

\section{Insulin Resistance and Cardiac Energy Metabolism}

Cardiomyocyte is a typical insulin-targeting cell. Chronic high fat intake promotes the development of insulin resistance in cardiomyocytes, leads to cardiometabolic dysfunction, and accelerates the development of left ventricular dysfunction and cardiac remodeling [84-86]. In diabetes, increased oxidation and storage of fatty 
acids in the heart can lead to diastolic dysfunction, which is also associated with insulin resistance [23]. In the case of insulin resistance, reduced IRS-1 tyrosine phosphorylation reduces the activation of PI3K-Akt signaling pathway and inhibits downstream insulin-mediated metabolic regulation [86]. The decrease of Akt phosphorylation directly leads to the decrease of GLUT4 translocation, which reduces the uptake of glucose in myocardium $[86,87]$. Reduced insulin signaling also inhibits glucose utilization by regulating the role of enzymes in the glycolysis pathway, such as hexokinase and phosphofructokinase $2[88,89]$. Declines in myocardial glucose uptake and utilization have also been observed in patients with diabetes [90].

There are many studies that suggest that excess lipid induces insulin resistance through DAG and ceramide [35, 74, 91, 92], which greatly affects energy metabolism. DAG has been shown to be more associated with myocardial insulin resistance than ceramides [36]. DAG-mediated PKC activation is currently recognized as the main cause of DAG-induced insulin resistance. PKC activation reduces tyrosine phosphorylation of IRS-1 resulting in reduced insulin-PI3K-Akt signaling [91]. Ceramide inhibits Akt activity, affects GLUT4 translocation and glucose uptake by activating $\mathrm{PKC} \zeta$ and protein phosphatase 2 (PP2A) [93, 94].

All in all, the insulin cascade forms a vicious circle, aggravating the heart's excessive dependence on fatty acids for energy and the acceleration of oxygen consumption. It has also been reported that, in addition to lipids, branched amino acids, iron overload can also lead to heart insulin resistance [95-97].

In addition to insulin, AMP-activated protein kinase (AMPK) also plays an important role in regulating substrate utilization. And insulin signal transduction are two relatively independent signaling pathways, but they also affect each other. The study showed that increased insulin sensitivity and increased glucose uptake and utilization were observed in mice with AMPK deletion [98]. Activated AMPK inhibits glycogen, fatty acid, and protein synthesis, and enhances glucose/fat uptake, mitochondrial metabolism, and autophagy by phosphorylating GLUT4/CD36, PGC-1 $\alpha /$ SIRT, and ACC2, respectively [99]. The interference of long-term excessive fatty acid level in the heart not only causes the change of PI3K-Akt-mediated insulin signaling, but also changes the AMPK-eNOS signaling. Although no studies have confirmed this, it can be inferred that FA can lead to cardiac energy metabolism disorders through AMPK even in the absence of insulin resistance.

\section{Concluding Remarks}

The heart needs a balanced ratio of fatty acids to glucose metabolism. When that balance is disrupted, it triggers a cascade of butterfly effects that imbalance the energy metabolism of the heart, resulting in impaired cardiac function and structure. The disturbance of myocardial energy metabolism and lipid toxicity caused by lipid overload seriously affects cardiac function. Chronic lipid overload can lead to heart failure. First, excess lipid promotes FAT/ CD36 uptake of fatty acids by increasing circulating free fatty acids through the action of insulin. Meanwhile, excessive intake of fatty acids up-regulated the expression of $\beta$-oxidation-related genes and enzymes and promoted the utilization of fatty acids. During this process, insulin resistance is induced by accumulating lipid toxicity, which inhibits glut4-mediated glucose uptake and utilization. The increase of fatty acid/glucose oxidation ratio resulted in myocardial over-dependence on fatty acid for energy and increased oxygen consumption. In addition to affecting energy metabolism, lipid toxicity and hypoxia cause mitochondrial damage and reduce mitochondrial efficiency.

By elucidating the pathological changes of the heart caused by lipid overload at the cellular and molecular levels, it is helpful to identify potential therapeutic targets. In addition to explaining the mechanisms of lipid overdose-induced cardiac injury, this review also mentions some factors leading to lipid toxicity, among which the role of PTMs of proteins has attracted increasing attention in recent years. These new descriptions may provide new ideas for cardiometabolic intervention.

\section{Authors' Contributions}

AY and GNX performed the literature review and wrote the manuscript. YW and LPG helped in the revision of the review. XYD conceived the review article. All authors approved the final version of the review.

\section{Funding Information}

This work was supported by Tianjin Postgraduate Research and Innovation Project (2020YJSB196).

\section{Conflict of Interest}

The authors declare that they have no conflict of interest.

\section{References}

[1] Faramawi MF, Delhey L, Abouelenein S et al. Metabolic syndrome and P-wave duration in the American population. Ann Epidemiol 2020; 46: 5-11

[2] Welsh C, Welsh P, Celis-Morales CA et al. Glycated hemoglobin, prediabetes, and the links to cardiovascular disease: data From UK biobank. Diabetes Care 2020; 43: 440-445

[3] Spence JD, Viscoli CM, Inzucchi SE et al. Pioglitazone therapy in patients with stroke and prediabetes: a post hoc analysis of the IRIS randomized clinical trial. JAMA Neurol 2019; 76: 526-535

[4] Sorensen BM, Houben AJ, Berendschot TT et al. Prediabetes and type 2 diabetes are associated with generalized microvascular dysfunction: the Maastricht study. Circulation 2016; 134: 1339-1352

[5] Rhee E], Kwon H, Park SE et al. Associations among obesity degree, glycemic status, and risk of heart failure in 9,720,220 Korean adults. Diabetes Metab J 2020; 44: 592-601

[6] Linssen PBC, Veugen MG], Henry RMA et al. Associations of (pre) diabetes with right ventricular and atrial structure and function: the Maastricht Study. Cardiovasc Diabetol 2020; 19: 88

[7] Kristensen SL, Preiss D, Jhund PS et al. Risk related to pre-diabetes mellitus and diabetes mellitus in heart failure with reduced ejection fraction: insights from prospective comparison of ARNI with ACEI to determine impact on global mortality and morbidity in heart failure trial. Circ Heart Fail 2016; 9 
[8] Selvin E, Lazo M, Chen $\mathrm{Y}$ et al. Diabetes mellitus, prediabetes, and incidence of subclinical myocardial damage. Circulation 2014; 130 : 1374-1382

[9] Burchfiel CM, Skelton TN, Andrew ME et al. Metabolic syndrome and echocardiographic left ventricular mass in blacks: the atherosclerosis risk in communities (ARIC) study. Circulation 2005; 112: 819-827

[10] Fontes-Carvalho R, Ladeiras-Lopes R, Bettencourt P et al. Diastolic dysfunction in the diabetic continuum: association with insulin resistance, metabolic syndrome and type 2 diabetes. Cardiovasc Diabetol 2015; 14: 4

[11] Jia G, Whaley-Connell A, Sowers JR. Diabetic cardiomyopathy: a hyperglycaemia- and insulin-resistance-induced heart disease. Diabetologia 2018; 61: 21-28

[12] Dillmann WH. Diabetic cardiomyopathy. Circ Res 2019; 124: 1160-1162

[13] Federico M, De la Fuente S, Palomeque J et al. The role of mitochondria in metabolic disease: a special emphasis on heart dysfunction. J Physiol 2021; 599: 3477-3493

[14] Gao Y, Ren Y, Guo YK et al. Metabolic syndrome and myocardium steatosis in subclinical type 2 diabetes mellitus: a (1) $\mathrm{H}$-magnetic resonance spectroscopy study. Cardiovasc Diabetol 2020; 19: 70

[15] Marfella R, Di Filippo C, Portoghese M et al. Myocardial lipid accumulation in patients with pressure-overloaded heart and metabolic syndrome. J Lipid Res 2009; 50: 2314-2323

[16] Costantino S, Akhmedov A, Melina G et al. Obesity-induced activation of JunD promotes myocardial lipid accumulation and metabolic cardiomyopathy. Eur Heart J 2019; 40: 997-1008

[17] Doenst T, Nguyen TD, Abel ED. Cardiac metabolism in heart failure: implications beyond ATP production. Circ Res 2013; 113: 709-724

[18] Horikoshi Y, Yan Y, Terashvili M et al. Fatty acid-treated induced pluripotent stem cell-derived human cardiomyocytes exhibit adult cardiomyocyte-like energy metabolism phenotypes. Cells 2019; 8:

[19] Bekhite MM, Gonzalez Delgado A, Menz F et al. Longitudinal metabolic profiling of cardiomyocytes derived from human-induced pluripotent stem cells. Basic Res Cardiol 2020; 115: 37

[20] Dolinsky VW, Dyck JR. Role of AMP-activated protein kinase in healthy and diseased hearts. Am J Physiol Heart Circ Physiol 2006; 291: H2557-H2569

[21] Neubauer S. The failing heart--an engine out of fuel. N Engl ] Med 2007; 356: 1140-1151

[22] Luiken J, Nabben M, Neumann D et al. Understanding the distinct subcellular trafficking of CD36 and GLUT4 during the development of myocardial insulin resistance. Biochim Biophys Acta Mol Basis Dis 2020; 1866: 165775

[23] Kerr M, Dodd MS, Heather LC. The 'Goldilocks zone' of fatty acid metabolism; to ensure that the relationship with cardiac function is just right. Clin Sci (Lond) 2017; 131: 2079-2094

[24] Lopaschuk GD, Ussher JR, Folmes CD et al. Myocardial fatty acid metabolism in health and disease. Physiol Rev 2010; 90: 207-258

[25] McGarry JD, Mannaerts GP, Foster DW. A possible role for malon$\mathrm{yl}-\mathrm{CoA}$ in the regulation of hepatic fatty acid oxidation and ketogenesis. J Clin Invest 1977; 60: 265-270

[26] Priesnitz C, Becker T. Pathways to balance mitochondrial translation and protein import. Genes Dev 2018; 32: 1285-1296

[27] Caruana NJ, Stroud DA. The road to the structure of the mitochondrial respiratory chain supercomplex. Biochem Soc Trans 2020; 48: $621-629$

[28] Mehdipour AR, Hummer G. Cardiolipin puts the seal on ATP synthase. Proc Natl Acad Sci U S A 2016; 113: 8568-8570

[29] Zhu B, Li MY, Lin Q et al. Lipid oversupply induces CD36 sarcolemmal translocation via dual modulation of PKCzeta and TBC1D1: an early event prior to insulin resistance. Theranostics 2020; 10: 1332-1354
[30] Glatz JF, Luiken JJ. From fat to FAT (CD36/SR-B2): Understanding the regulation of cellular fatty acid uptake. Biochimie 2017; 136: 21-26

[31] Ouwens DM, Diamant M, Fodor M et al. Cardiac contractile dysfunction in insulin-resistant rats fed a high-fat diet is associated with elevated CD36-mediated fatty acid uptake and esterification. Diabetologia 2007; 50: 1938-1948

[32] Angin Y, Steinbusch LK, Simons P] et al. CD36 inhibition prevents lipid accumulation and contractile dysfunction in rat cardiomyocytes. Biochem J 2012; 448: 43-53

[33] Bonen A, Jain SS, Snook LA et al. Extremely rapid increase in fatty acid transport and intramyocellular lipid accumulation but markedly delayed insulin resistance after high fat feeding in rats. Diabetologia 2015; 58: 2381-2391

[34] Renguet E, Bultot L, Beauloye $C$ et al. The regulation of insulin-stimulated cardiac glucose transport via protein acetylation. Front Cardiovasc Med 2018; 5: 70

[35] Chaurasia B, Tippetts TS, Mayoral Monibas R et al. Targeting a ceramide double bond improves insulin resistance and hepatic steatosis. Science 2019; 365: 386-392

[36] Zhang L, Ussher JR, Oka T et al. Cardiac diacylglycerol accumulation in high fat-fed mice is associated with impaired insulin-stimulated glucose oxidation. Cardiovasc Res 2011; 89: 148-156

[37] Sung MM, Byrne NJ, Kim TT et al. Cardiomyocyte-specific ablation of CD36 accelerates the progression from compensated cardiac hypertrophy to heart failure. Am J Physiol Heart Circ Physiol 2017; 312: H552-H560

[38] Glatz JFC, Nabben M, Young ME et al. Re-balancing cellular energy substrate metabolism to mend the failing heart. Biochim Biophys Acta Mol Basis Dis 2020; 1866: 165579

[39] Wang S, Schianchi F, Neumann D et al. Specific amino acid supplementation rescues the heart from lipid overload-induced insulin resistance and contractile dysfunction by targeting the endosomal mTOR-v-ATPase axis. Mol Metab 2021; 53: 101293

[40] Kim G, Jo K, Kim KJ et al. Visceral adiposity is associated with altered myocardial glucose uptake measured by (18)FDG-PET in 346 subjects with normal glucose tolerance, prediabetes, and type 2 diabetes. Cardiovasc Diabetol 2015; 14: 148

[41] Cortassa S, Caceres V, Tocchetti CG et al. Metabolic remodelling of glucose, fatty acid and redox pathways in the heart of type 2 diabetic mice. J Physiol 2020; 598: 1393-1415

[42] Belke DD, Larsen TS, Gibbs EM et al. Altered metabolism causes cardiac dysfunction in perfused hearts from diabetic $(\mathrm{db} / \mathrm{db})$ mice. Am J Physiol Endocrinol Metab 2000; 279: E1104-E1113

[43] Fukushima A, Lopaschuk GD. Cardiac fatty acid oxidation in heart failure associated with obesity and diabetes. Biochim Biophys Acta 2016; 1861: 1525-1534

[44] Carpentier AC. Abnormal myocardial dietary fatty acid metabolism and diabetic cardiomyopathy. Can J Cardiol 2018; 34: 605-614

[45] Cole MA, Murray AJ, Cochlin LE et al. A high fat diet increases mitochondrial fatty acid oxidation and uncoupling to decrease efficiency in rat heart. Basic Res Cardiol 2011; 106: 447-457

[46] Finck BN, Lehman JJ, Leone TC et al. The cardiac phenotype induced by PPARalpha overexpression mimics that caused by diabetes mellitus. J Clin Invest 2002; 109: 121-130

[47] Dyck JR, Cheng JF, Stanley WC et al. Malonyl coenzyme a decarboxylase inhibition protects the ischemic heart by inhibiting fatty acid oxidation and stimulating glucose oxidation. Circ Res 2004; 94 : e78-e84

[48] Donelson J, Wang Q, Monroe TO et al. Cardiac-specific ablation of glutaredoxin 3 leads to cardiac hypertrophy and heart failure. Physiol Rep 2019; 7: e14071 
[49] Lillig CH, Berndt C, Holmgren A. Glutaredoxin systems. Biochim Biophys Acta 2008; 1780: 1304-1317

[50] Cheng N, Mo Q, Donelson J et al. Crucial role of mammalian glutaredoxin 3 in cardiac energy metabolism in diet-induced obese mice revealed by transcriptome analysis. Int J Biol Sci 2021; 17: 2871-2883

[51] Ying F, Liu H, Ching Tang EH et al. Prostaglandin E receptor subtype 4 protects against diabetic cardiomyopathy by modulating cardiac fatty acid metabolism via FOXO1/CD36 signalling. Biochem Biophys Res Commun 2021; 548: 196-203

[52] Mishra P, Chan DC. Metabolic regulation of mitochondrial dynamics. J Cell Biol 2016; 212: 379-387

[53] Koncsos G, Varga ZV, Baranyai T et al. Diastolic dysfunction in prediabetic male rats: Role of mitochondrial oxidative stress. Am J Physiol Heart Circ Physiol 2016; 311: H927-H943

[54] Sverdlov AL, Elezaby A, Qin F et al. Mitochondrial reactive oxygen species mediate cardiac structural, functional, and mitochondrial consequences of diet-induced metabolic heart disease. J Am Heart Assoc 2016; 5:

[55] Boudina S, Sena S, Theobald $\mathrm{H}$ et al. Mitochondrial energetics in the heart in obesity-related diabetes: direct evidence for increased uncoupled respiration and activation of uncoupling proteins. Diabetes 2007; 56: 2457-2466

[56] Boudina S, Bugger $\mathrm{H}$, Sena $\mathrm{S}$ et al. Contribution of impaired myocardial insulin signaling to mitochondrial dysfunction and oxidative stress in the heart. Circulation 2009; 119: 1272-1283

[57] Belke DD, Betuing S, Tuttle M] et al. Insulin signaling coordinately regulates cardiac size, metabolism, and contractile protein isoform expression. J Clin Invest 2002; 109: 629-639

[58] Maneechote C, Palee S, Apaijai N et al. Mitochondrial dynamic modulation exerts cardiometabolic protection in obese insulin-resistant rats. Clin Sci (Lond) 2019; 133: 2431-2447

[59] Waddingham MT, Sonobe T, Tsuchimochi $\mathrm{H}$ et al. Diastolic dysfunction is initiated by cardiomyocyte impairment ahead of endothelial dysfunction due to increased oxidative stress and inflammation in an experimental prediabetes model. J Mol Cell Cardiol 2019; 137: $119-131$

[60] Stacchiotti A, Favero G, Giugno L et al. Melatonin efficacy in obese leptin-deficient mice heart. Nutrients 2017; 9:

[61] Gottlieb RA, Gustafsson AB. Mitochondrial turnover in the heart. Biochim Biophys Acta 2011; 1813: 1295-1301

[62] Gao S, Hu J. Mitochondrial fusion: the machineries in and out. Trends Cell Biol 2021; 31: 62-74

[63] Filadi R, Pendin D, Pizzo P. Mitofusin 2: from functions to disease. Cell Death Dis 2018; 9: 330

[64] Tezze C, Romanello V, Desbats MA et al. Age-associated loss of OPA1 in muscle impacts muscle mass, metabolic homeostasis, systemic inflammation, and epithelial senescence. Cell Metab 2017; 25: 1374-1389 e1376

[65] Elezaby A, Sverdlov AL, Tu VH et al. Mitochondrial remodeling in mice with cardiomyocyte-specific lipid overload. J Mol Cell Cardiol 2015; 79: $275-283$

[66] Hu Q, Zhang H, Gutierrez Cortes N et al. Increased Drp1 acetylation by lipid overload induces cardiomyocyte death and heart dysfunction. Circ Res 2020; 126: 456-470

[67] Tsushima K, Bugger H, Wende AR et al. Mitochondrial reactive oxygen species in lipotoxic hearts induce post-translational modifications of AKAP121, DRP1, and OPA1 that promote mitochondrial fission. Circ Res 2018; 122: 58-73

[68] Schaffer JE. Lipotoxicity: when tissues overeat. Curr Opin Lipidol 2003; 14: 281-287
[69] Nakamura M, Liu T, Husain S et al. Glycogen synthase kinase-3alpha promotes fatty acid uptake and lipotoxic cardiomyopathy. Cell Metab 2019; 29: 1119-1134 e1112

[70] Roe ND, Handzlik MK, Li T et al. The role of diacylglycerol acyltransferase (DGAT) 1 and 2 in cardiac metabolism and function. Sci Rep 2018; 8: 4983

[71] Peterson LR, Xanthakis V, Duncan MS et al. Ceramide remodeling and risk of cardiovascular events and mortality. J Am Heart Assoc 2018; 7 :

[72] Ljubkovic M, Gressette M, Bulat C et al. Disturbed fatty acid oxidation, endoplasmic reticulum stress, and apoptosis in left ventricle of patients with type 2 diabetes. Diabetes 2019; 68: 1924-1933

[73] de la Monte SM, Tong M, Nguyen V et al. Ceramide-mediated insulin resistance and impairment of cognitive-motor functions. J Alzheimers Dis 2010; 21: 967-984

[74] Summers SA, Chaurasia B, Holland WL. Metabolic messengers: ceramides. Nat Metab 2019; 1: 1051-1058

[75] Blair HC, Sepulveda J, Papachristou DJ. Nature and nurture in atherosclerosis: the roles of acylcarnitine and cell membrane-fatty acid intermediates. Vascul Pharmacol 2016; 78: 17-23

[76] Pande SV, Blanchaer MC. Reversible inhibition of mitochondrial adenosine diphosphate phosphorylation by long chain acyl coenzyme A esters. J Biol Chem 1971; 246: 402-411

[77] Ciapaite J, Van Eikenhorst G, Bakker S] et al. Modular kinetic analysis of the adenine nucleotide translocator-mediated effects of palmitoyl-CoA on the oxidative phosphorylation in isolated rat liver mitochondria. Diabetes 2005; 54: 944-951

[78] Kerr M, Dennis K, Carr CA et al. Diabetic mitochondria are resistant to palmitoyl CoA inhibition of respiration, which is detrimental during ischemia. FASEB J 2021; 35: e21765

[79] Schianchi F, Glatz JFC, Navarro Gascon A et al. Putative role of protein palmitoylation in cardiac lipid-induced insulin resistance. Int J Mol Sci 2020; 21:

[80] Dasgupta S, Bhattacharya S, Maitra S et al. Mechanism of lipid induced insulin resistance: activated PKCepsilon is a key regulator. Biochim Biophys Acta 2011; 1812: 495-506

[81] Zhao L, Zhang C, Luo X et al. CD36 palmitoylation disrupts free fatty acid metabolism and promotes tissue inflammation in non-alcoholic steatohepatitis. J Hepatol 2018; 69: 705-717

[82] Sundaresan NR, Pillai VB, Wolfgeher D et al. The deacetylase SIRT1 promotes membrane localization and activation of Akt and PDK1 during tumorigenesis and cardiac hypertrophy. Sci Signal 2011; 4: ra46

[83] Alrob OA, Sankaralingam S, Ma C et al. Obesity-induced lysine acetylation increases cardiac fatty acid oxidation and impairs insulin signalling. Cardiovasc Res 2014; 103: 485-497

[84] Aurigemma GP, de Simone G, Fitzgibbons TP. Cardiac remodeling in obesity. Circ Cardiovasc Imaging 2013; 6: 142-152

[85] Apaijai N, Arinno A, Palee S et al. High-saturated fat high-sugar diet accelerates left-ventricular dysfunction faster than high-saturated fat diet alone via increasing oxidative stress and apoptosis in obese-insulin resistant rats. Mol Nutr Food Res 2019; 63: e1800729

[86] Han L, Liu J, Zhu L et al. Free fatty acid can induce cardiac dysfunction and alter insulin signaling pathways in the heart. Lipids Health Dis 2018; 17: 185

[87] Wang Z, Wang Y, Han Y et al. Akt is a critical node of acute myocardial insulin resistance and cardiac dysfunction after cardiopulmonary bypass. Life Sci 2019; 234: 116734

[88] Bockus LB, Matsuzaki S, Vadvalkar SS et al. Cardiac insulin signaling regulates glycolysis through phosphofructokinase 2 content and activity. J Am Heart Assoc 2017; 6: 
[89] Gejl M, Sondergaard HM, Stecher C et al. Exenatide alters myocardial glucose transport and uptake depending on insulin resistance and increases myocardial blood flow in patients with type 2 diabetes. J Clin Endocrinol Metab 2012; 97: E1165-E1169

[90] Sondergaard HM, Bottcher M, Marie Madsen M et al. Impact of type 2 diabetes on myocardial insulin sensitivity to glucose uptake and perfusion in patients with coronary artery disease. J Clin Endocrinol Metab 2006; 91: 4854-4861

[91] Yu C, Chen Y, Cline GW et al. Mechanism by which fatty acids inhibit insulin activation of insulin receptor substrate-1 (IRS-1)-associated phosphatidylinositol 3-kinase activity in muscle. J Biol Chem 2002; 277: 50230-50236

[92] Wali JA, Jarzebska N, Raubenheimer D et al. Cardio-metabolic effects of high-fat diets and their underlying mechanisms - a narrative review. Nutrients 2020; 12:

[93] Summers SA, Garza LA, Zhou H et al. Regulation of insulin-stimulated glucose transporter GLUT4 translocation and Akt kinase activity by ceramide. Mol Cell Biol 1998; 18: 5457-5464
[94] Chen TC, Benjamin DI, Kuo T et al. The glucocorticoid-Angpt|4-ceramide axis induces insulin resistance through PP2A and PKCzeta. Sci Signal 2017; 10:

[95] Uddin GM, Zhang L, Shah S et al. Impaired branched chain amino acid oxidation contributes to cardiac insulin resistance in heart failure. Cardiovasc Diabetol 2019; 18: 86

[96] Sung HK, Song E, Jahng JWS et al. Iron induces insulin resistance in cardiomyocytes via regulation of oxidative stress. Sci Rep 2019; 9: 4668

[97] Neinast MD, Jang C, Hui S et al. Quantitative analysis of the whole-body metabolic fate of branched-chain amino acids. Cell Metab 2019; 29: 417-429 e414

[98] Lantier L, Williams AS, Williams IM et al. Reciprocity between skeletal muscle AMPK deletion and insulin action in diet-induced obese mice. Diabetes 2020; 69: 1636-1649

[99] Ke R, Xu Q, Li C et al. Mechanisms of AMPK in the maintenance of ATP balance during energy metabolism. Cell Biol Int 2018; 42: 384-392 